\title{
The use of focus groups in evaluating quality of life components among elderly Chinese people
}

\author{
Kai-Kuen Leung ${ }^{1}$, En-Chang $\mathrm{Wu}^{2}$, Bee-Horng Lue ${ }^{1,3}$ \& Li-Yu Tang ${ }^{4}$ \\ ${ }^{1}$ Department of Family Medicine, National Taiwan University Hospital (E-mail: kkleung@ha.mc.ntu. \\ edu.tw); ${ }^{2}$ School of Psychology, National Taiwan University College of Science; ${ }^{3}$ Department of Social \\ Medicine, National Taiwan University College of Medicine; ${ }^{4}$ College of Nursing, Yang Ming University
}

Accepted in revised form 20 December 2002

\begin{abstract}
In Taiwan, to measure the quality of life (QOL) of elderly Chinese, one must rely on instruments developed in other Chinese or Western populations and not specifically for the elderly. The purpose of this study is to understand the components of QOL for elderly Chinese from Taiwan living in residential homes or in their communities. Forty-four elderly men and women divided into six focus groups were interviewed on video tape and the resultant recording was analyzed qualitatively by six independent researchers. The study yielded 15 QOL domains grouped into six dimensions: physical health (physical well-being, impact of illness, medical care), psychological health (mood states, life attitude and retrospection, philosophy of living, self-efficacy), social function (connectedness, exercise and leisure activities, social activities and services), living environment (living environment and arrangements, institutional factors), economic status, and religion and death (religion, death). For elderly Chinese in Taiwan, positive and negative life domains are equally important in the perception of life quality; person-environment interaction is a major consideration in the evaluation of QOL; family ties are an important component of QOL; traditional Chinese beliefs exert a positive influence on perceived QOL; and social functioning and vitality have a different meaning in Chinese compared to Western cultures.
\end{abstract}

Key words: Chinese, Elderly, Focus group interview, Quality of life

\section{Introduction}

Quality of life (QOL) is an important health index for the elderly, especially for those with chronic diseases or disability. Unfortunately, a review of the literature reveals that there are few, if any, instruments developed specifically to measure the QOL of the elderly [1-3] who have different needs and values from younger adults [3]. To measure QOL in elderly Chinese from Taiwan using the existing instruments, an investigator would need to rely on a questionnaire developed with Western populations and translated into Taiwanese [4], Mandarin [5] or Cantonese [6], or a questionnaire developed for the Chinese from Mainland China
[7-10], America [11], or Hong Kong [12, 13] and not specifically for use with elderly samples.

The Chinese are a heterogeneous, widely dispersed people. Mainland and Hong Kong Chinese people have different life experiences, life styles, environments, customs, communication style, and in some cases, language and dialect, from the Chinese living in Taiwan [14]. Yet despite geographic distance, the Chinese also share some common cultural threads and values. These differences and similarities influence the domains of their QOL. Therefore, the purpose of this study is to describe the domains and dimensions of the QOL of elderly Chinese people from Taiwan. To achieve this we used focus group interviews (FGI) among those 
living in residential homes or those who remained living in their communities. The long-term goal is to develop a valid, reliable, and sensitive measure of QOL of elderly Chinese from Taiwan.

\section{Background}

A review of the relevant literature reveals that there have been two types of QOL research done among Chinese people. The first was the translation and transcultural adaptation of QOL questionnaires developed in Western countries such as the Chinese version of the Functional Assessment of Cancer Therapy-General (FACT-G) [15], the diabetes QOL measure [16], the EORTC QLQC30 [5] and the Short Form 36 Health Survey (SF-36) [11]. Several transcultural differences were found among these studies. In the FACT-G questionnaire, intimacy between close partners is less related to sexual relations among Chinese populations than in the West. Chinese culture stresses familial obligations more than individual needs [15], and social support provided by family members is more important than support from other sources [17]. Moreover, talking about sex openly is not appropriate in Chinese culture. According to two studies among older Hong Kong Chinese, Chinese and Western patients had different responses to chronic illnesses $[16,18]$. Researchers found that for the elderly Hong Kong Chinese, to be 'physically ill' was regarded as an item in the worry scale instead of in the hypothesized impact scale [16]. A lack of adverse effects of diabetes mellitus and heart disease on the healthrelated QOL of Chinese patients suggest that Chinese culture may promote positive adaptation to chronic illnesses [18]. The Short Form 36 Health Survey (SF-36) has been translated into three Chinese versions: the US version [11], the Hong Kong version [6], and the Taiwan version [4]. Together they provide valuable information for transcultural and transsocietal comparison. In the US study, researchers found that there were two social functioning items on the SF-36 that correlated with the vitality and mental health scales more than with their own scale [11]. Collectivism ideology in Chinese culture may explain the denial of disturbance of emotion and physical health on social activities. Moreover, a correlation between vitality and mental health was observed in several studies $[11,19,20]$. All these studies of Chinese Americans suggest that 'vitality' might be perceived differently by Chinese and Western people. For the Chinese, vitality means mental strength rather than physical strength. Lack of vitality usually means lack of spirit or 'Yuan-Chi' (literally 'vital air'). In a study of middle-aged women in Kinmen, Taiwan, researchers found that the vitality and social functioning scales were more associated with mental health than physical health. Bodily pain was associated with mental health in this study. This result was not reported in the Hong Kong and US studies, and suggests a somatization tendency among elderly Taiwanese [4].

Not all studies revealed different results between Chinese and Western people. Researchers found no difference in the QOL structure between the original and translated Chinese version of the EORTC QLQ-C30. However, this result may be biased by the small sample size and by the arbitrary recruitment of only patients with gynecological cancer [5].

The second type of QOL research was the development of QOL questionnaires primarily aimed towards Chinese societies. Examples of these are the Inventory of Life Quality of the Elderly (ILQE) [7] and the Quality of Life Inventory (QOL-I) from Mainland China [9], and the health status indicator (HIS) from Hong Kong [12]. A study using the ILQE with elderly Mainland Chinese revealed that two main factors, income and family harmony, explained $90 \%$ of the total variance in the study population [7]. Another study done on elderly hypertensive workers revealed that physical status and functional daily activities were major determinants of QOL [8].

The QOL-I is an instrument developed for use in the general population in a community [9]. An interesting finding was that all objective QOL indicators declined with age, but all subjective QOL indicators except for physical function increased with age [10].

In the HIS, QOL is classified into seven areas and arranged in a hierarchy under the physical and psychological categories. However, how these seven areas were obtained was not clearly explained by the researchers [12].

In Taiwan, the majority of the population is descendants of people who came from the Fu-Kien 
province in Mainland China. Although Mandarin is the official language, many people, especially older people, use the Taiwanese dialect. Furthermore, people in Taiwan live a more westernized life style than people in Mainland China. Socioeconomic factors in different Chinese societies may have some influence on the QOL of elderly people. With the industrialization of Taiwanese society, many elderly people are cared for in institutions rather than in their homes. A government survey showed that only $0.8 \%$ of the elderly aged 65 years old and over, were institutionalized in 1987 [21]. However, the percentage rose to $1.2 \%$ in 1991 [22] and has grown rapidly in the past 10 years. An institution is an environment entirely different from one's own household. The QOL of the institutionalized elderly is a concern for health policy makers and health care givers. Thus, this study sought to address the components of QOL as expressed by the elderly living in residential homes in comparison to those who remained in their communities.

\section{Methods}

Our study relied on FGI to gather information directly from our informants about perceptions of QOL among the elderly Taiwanese living either in their own community or in residential homes. The FGI is a qualitative research technique used to obtain data about feelings and opinions from a small group of participants about a given problem, experience, service or other phenomenon [23]. An FGI can have three different approaches: exploratory, clinical, and phenomenological [24]. The exploratory approach is more appropriate for collecting prescientific knowledge, pilot testing, generating constructs and hypotheses, and testing them against everyday experience. This approach is also suitable for exploring the perceptions of QOL in elderly people.

\section{Composition of groups}

A purposive sample of elderly people living in a residential home and a semi-urban community in Taipei County was chosen to participate in the focus groups. Factors such as sex, residence, and spoken language were considered in the composition of each group to provide homogeneity within groups and diversity between groups. A total of 44 elderly people divided into six focus groups participated: two groups of men from the residential home, two groups of women from the residential home, a group of men from the community, and a group of women from the community. Each group from the residential home had seven participants and each group from the community had eight participants.

All groups from the community and one men's group and one women's group from the residential home spoke the Taiwanese language. The other two groups from the residential home spoke Mandarin. Although nearly all elderly subjects had one or more chronic medical conditions, on average they were in good physical condition and could perform daily activities without assistance. The sociodemographic data of the elderly subjects are summarized in Table 1.

Subjects who spoke the Taiwanese language were born in Taiwan. Those who spoke Mandarin were born in Mainland China and came to Taiwan after the civil war before 1949. Residents of the residential home had lived there between 2 and 11 years. Each group was led by two clinical psychologists (one moderator and one co-moderator) who had been trained and had experience in leading focus groups. Six clinical psychologists (five men and one woman) participated in this study. Each psychologist was the moderator of one group and the co-moderator of another group.

Table 1. Sociodemographic characteristics of elderly people in the focus groups

\begin{tabular}{ll}
\hline Age & $\begin{array}{l}\text { Mean 75.41 } \\
\text { range 65-86 5.71 years }\end{array}$ \\
\hline Gender & \\
$\quad$ Men & $22(50.0)$ \\
Women & $22(50.0)$ \\
Residence & \\
Community & $14(31.8)$ \\
Institution & $30(68.2)$ \\
Education & \\
College & $8(18.2)$ \\
High school & $15(34.1)$ \\
Middle school & $13(29.5)$ \\
No formal education & $8(18.2)$ \\
\hline
\end{tabular}

Percentage is shown in parentheses. 
Moderators and co-moderators were randomly assigned to each group and did not know the group members before the interview. During the group interview, an observer sat at the back of the group to observe and take notes on opinions and issues raised in the group and the interactive process of the group.

\section{Process of the focus group interview}

Each focused interview began with a short warmup period of about $10-15 \mathrm{~min}$. The moderator and co-moderator welcomed the participants and introduced the members of the group. During the introduction, we found that although some group members already knew each other, no group members were close friends or relatives, and their interaction in the group was not affected by their relationships. A structuring session followed the warm-up period. The moderator explained the mission of the group and obtained an oral consent from the participants. Each group met only once and the amount of time for the interview was about one and a half to 2 hours. During the interview, the moderator's responsibilities were to create a non-threatening and comfortable environment, to facilitate group interactions, probe comments, cover important topics in the prepared outline by asking open-ended questions, encourage involvement and response, and control the allocation of time. Open-ended questions asked in the groups included: 'Are you satisfied with the quality of your life? Why?', 'What are the most important things in your life?', 'What things give elderly people life quality?', 'What do you need in order to make your life quality better?' The co-moderator helped the moderator to lead the group, took notes, reminded the moderator about the topics in the prepared outline, acted as time keeper, noted the formation of subgroups in the discussion, reflected the issues of individuals or subgroups to the whole group, and took the role of moderator if the moderator was engaged in solving an individual issue. Each FGI ended with a short closure wherein the moderators summarized the major opinions obtained and verified them with the participants. A debriefing between the moderator, co-moderator and observer occurred immediately after each interview. Preliminary findings and the group process were discussed. All group processes were recorded by two videocameras from different angles and two tape recorders.

\section{Analysis of data}

Tape-based analysis [25] was applied in this study. Six reviewers participated in the data analysis. Each focus group data was reviewed independently by two reviewers - one reviewer who participated and one reviewer who did not participate in that group. Field notes and records from the debriefing at the end of each focus group were also reviewed. Each reviewer wrote a transcript of all statements and issues related to QOL identified from the tapes. A series of six consensus meetings including all reviewers was held to analyze each focus group's data. In the meeting, the transcripts and field notes were compared and discussed. Information was first grouped into six dimensions, physical health, psychological health, social function, living environment, economic status, and religion and death. Within each dimension, information carrying different meanings was sorted out and named according to the various meanings. Videotapes were reviewed and discussed if any disagreement was encountered during the consensus meeting or the reviewers found that more information (especially non-verbal cues) was necessary for the analysis. A final consensus was reached with full agreement of all six reviewers in the consensus meeting.

\section{Results}

Fifteen QOL domains grouped into six dimensions were identified from the FGI. Table 2 summarizes the QOL domains and dimensions expressed by the elderly Chinese from Taiwan living in either residential homes or community settings; they also provide examples of conversation. These have been translated into English while maintaining the meaning of the original.

\section{Physical health}

Table 2 shows the three domains in the physical health dimension. 'Physical well-being' implies physical functions such as eating, sleeping, visual 


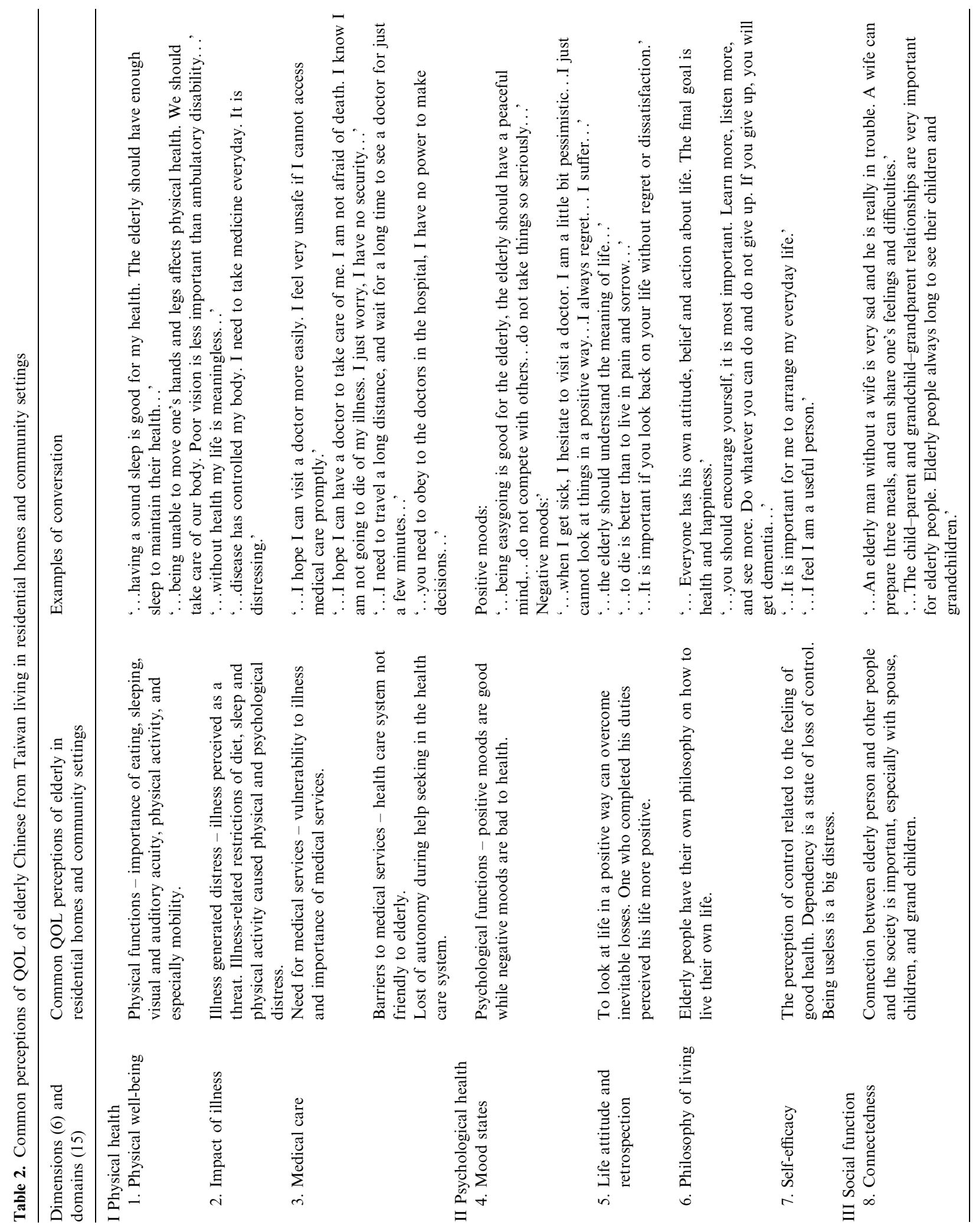




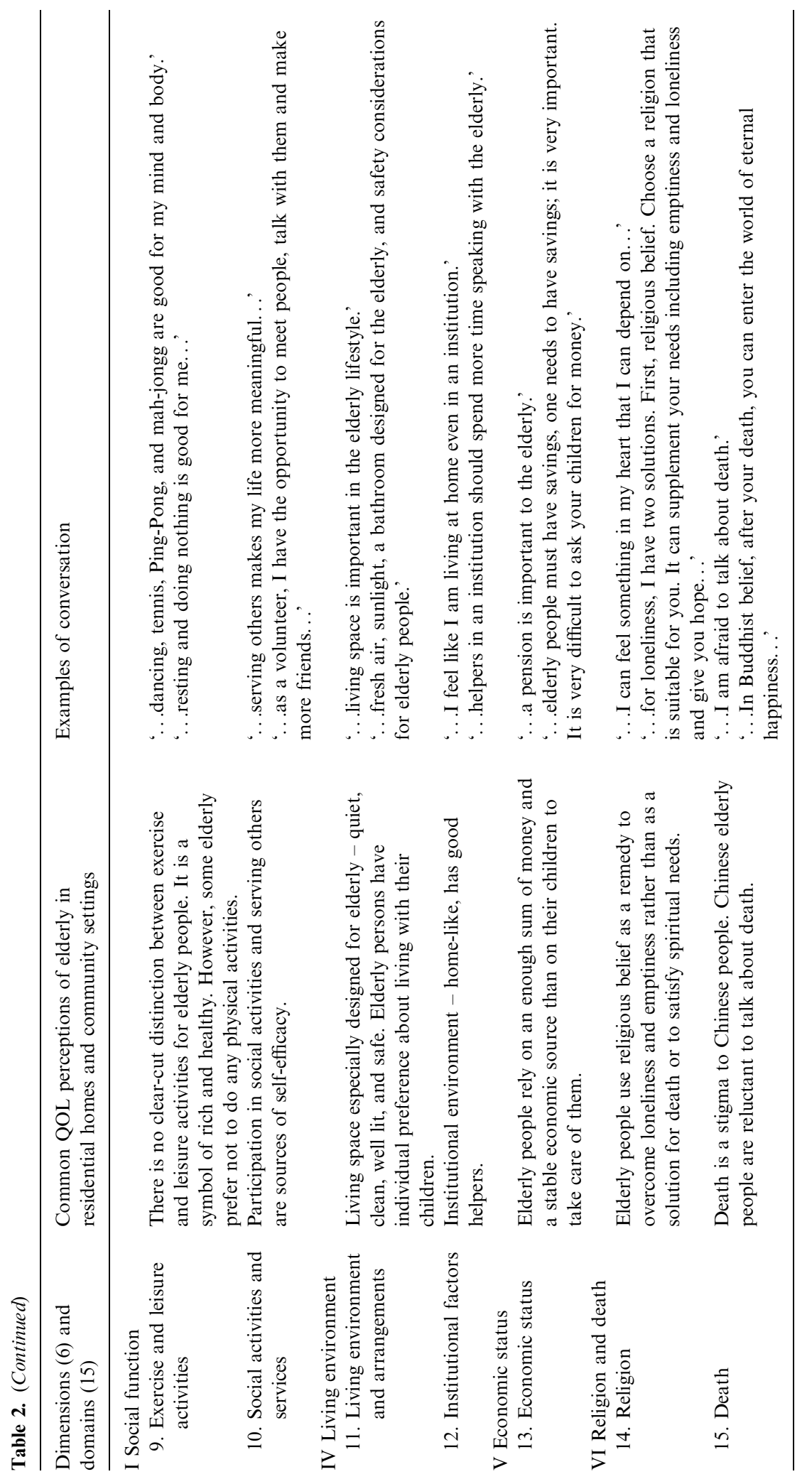


and auditory acuity, mobility, and physical activity. 'Impact of illness' implies the distress caused by chronic medical diseases. 'Medical care' includes the demands of medical service, barriers to receive medical care, and lost autonomy when seeking help in the health care system.

\section{Psychological health}

The psychological health dimension includes four domains (Table 2): 'Mood states' implies the psychological aspect of the elderly people. It can be divided into two opposite aspects, positive and negative moods. 'Life attitude and retrospection' implies the value and meaning that the elderly give to their lives. 'Philosophy of living' is the wisdom behind how the elderly live their own lives. 'Selfefficacy' implies the ability to control one's daily activities and to arrange one's daily schedule.

\section{Social function}

The social function dimension includes three domains (Table 2): 'Connectedness' implies the relationship between an elderly person and other people or society as a whole. Their relationships with their spouse, children and grandchildren were found to be important. Compared to kinship, friendship seemed to be a second choice for elderly people. 'Exercise and leisure activities' were activities for fun or for good health. Being able to do regular exercise or leisure activities was an indicator of the rich and healthy. 'Social activity and service' refers to their participation in social activities and to serving others.

\section{Living environment}

The living environment dimension includes two domains (Table 2): 'Living environment and arrangements' implies preferences of physical environments, persons in a household, and accessibility to relatives and friends. 'Institutional factors' are environment, facilities, and care provided by institutions.

\section{Economic status}

Economic status is a very important issue raised in the FGI (Table 2). Having enough money to use for the rest of their life became a basic requirement for life quality. Moreover, having a stable economic source was as important as quantity.

\section{Religion and death}

Information about religious belief and death are included in the last dimension (Table 2). In the focus interview, we failed to find evidence that elderly people used religion as a solution for the fear of death or to satisfy spiritual needs. Rather, elderly people seemed to use religious belief as a remedy to overcome loneliness and emptiness. The elderly did not seem to be distressed when talking about death in general. However, they did not want to talk more about the issue in the FGI.

Table 3 summarizes the different perceptions of QOL of Chinese from Taiwan living in residential homes and in community settings. Most of the differences arose from the dimensions of social function and living environment.

In this study, most Mandarin speaking elderly persons lived in institutions and most of the Taiwanese speaking elderly persons lived in their own communities. Examining elderly people from the same living environment did not reveal any difference between those who spoke Mandarin or Taiwanese.

A gender difference was also noted in this study. Old women talked more about their family and children and old men talked more about their friends and concerns about society. The relationship between husband and wife was frequently mentioned by the men but not by the women.

\section{Discussion}

This discussion focuses on the major findings in this study of QOL, and compares them with QOL studies in Western countries, Mainland China and Hong Kong. We will also consider differences between community-based and institutionalized elderly and the shortcomings of this study.

In our study, we identified 15 QOL domains. Of these, physical function, psychological distress, self-control, social function, economic factors, and perceived health have frequently been mentioned in the literature [26]. The impact of disease has been regarded as a proxy of QOL by some 
Table 3. Different perceptions of QOL of elderly Chinese from Taiwan living in residential homes vs. community settings

\begin{tabular}{|c|c|c|}
\hline Dimensions (6) and domains (15) & $\begin{array}{l}\text { Elderly in residential home } \\
\text { examples of conversation }\end{array}$ & $\begin{array}{l}\text { Elderly in community settings } \\
\text { examples of conversation }\end{array}$ \\
\hline \multicolumn{3}{|l|}{ III Social function } \\
\hline 8. Connectedness & $\begin{array}{l}\text { Emphasized friendship as well as } \\
\text { kinship } \\
\text { '...old friends are very important } \\
\text { to the elderly. If you have friends, } \\
\text { you have someone to talk to...' } \\
\text { Emphasized self needs } \\
\text {... an old man must take good } \\
\text { care of himself, no one will } \\
\text { responsible for you...' }\end{array}$ & $\begin{array}{l}\text { Emphasized relation with spouse and } \\
\text { other family members } \\
\text { '... the child-parent and grandchild } \\
\text {-grandparent relationships are very } \\
\text { important for elderly people.' } \\
\text { Emphasized family needs } \\
\text { '... my life has no regret if my } \\
\text { children can have a good career ...' }\end{array}$ \\
\hline $\begin{array}{l}\text { 9. Exercise and leisure } \\
\text { activities }\end{array}$ & $\begin{array}{l}\text { Reported more leisure activities } \\
\text {....here we have activities like } \\
\text { dancing, Karaoke, Tai-chi, drawing. .. }\end{array}$ & $\begin{array}{l}\text { Reported less leisure activities except } \\
\text { traveling } \\
\text { '...I always travel abroad with other } \\
\text { elderly in my community.' } \\
\text { '...traveling is fun, I have visited } \\
\text { many countries in the world...' }\end{array}$ \\
\hline \multicolumn{3}{|l|}{ IV Living environment } \\
\hline $\begin{array}{l}\text { 11. Living environment } \\
\text { and arrangement }\end{array}$ & $\begin{array}{l}\text { Emphasized facilities of the } \\
\text { institution } \\
\text { '... institution should have good } \\
\text { environments such as a place to } \\
\text { do exercise, medical facilities and } \\
\text { transportation service...' }\end{array}$ & $\begin{array}{l}\text { Emphasized accessibility to } \\
\text { relatives and friends } \\
\text { '... I prefer to live in a community } \\
\text { close to my children and friends, so } \\
\text { that I can visit them frequently...' }\end{array}$ \\
\hline 12. Institutional factors & $\begin{array}{l}\text { Staffs of an institution are } \\
\text { important to elderly people } \\
\text { '... helpers in an institution } \\
\text { should spend more time speaking } \\
\text { with the elderly...' }\end{array}$ & \\
\hline
\end{tabular}

researchers [27]. It was also a major topic across all focus groups in our study. Moreover, we found that Taiwanese elderly people worried about the consequences of physical illnesses, but were also concerned about the availability and affordability of medical care. Quality medical care seemed to be a basic need for the elderly in all the focus groups and was considered as a separate domain. This important finding requires further study.

During all the interviews, psychological needs received more attention than physical abilities. It was unlikely to be a bias caused by the moderators since we did not observe any guiding behavior directed to psychosocial issues in the groups. It was suggested that there is a hierarchical structure in the content of QOL from biological to social complexity [28]. Basic needs such as physical functions have to be satisfied before one can proceed to meet higher level needs. It was possible that the elderly people recruited in our study had their basic needs met. Social activity and service (i.e. helping others) as a QOL domain has rarely been mentioned in the literature. In our study, engaging in social activities as a volunteer to help others seemed to have a positive effect on many elderly people. These people seemed to be more active, confident and physically competent and they enjoyed their work. It is very possible that being able to help others can strengthen the selfperception of competency, and the helping process may also give elderly people a sense of fulfillment and self-satisfaction. In this study, the meaning of social service (helping others) was different from that of social functioning (interacting with others) in the SF-36.

Self-efficacy is defined as an individual's assessment of his or her ability to perform in specific situations [29]. In our study, the ability to perform activities and be independent of others was important to the perception of life quality. This result 
supports previous research which found that selfefficacy influenced health behavior, which resulted in a better health-related QOL [30].

The relationship with spouse, children, grandchildren, and friends contributes to good QOL in elderly people. The death of a spouse is the most stressful event in the elderly, especially elderly men. This finding is compatible with a previous study in America [31] and a cross-cultural study in the United States and India [32]. The connection between the marital relationship and subjective wellbeing seems to be universally consistent. In the ILQE, married life, child obedience, and family harmony are the three domains of QOL in elderly people. The emphasis on family and relationships among family members may be the most obvious difference in the relative value of QOL domains between Western and Chinese elderly from Taiwan.

Life attitudes and beliefs is another domain not measured in most QOL instruments. In this study, we found that the elderly have their own attitudes and beliefs about human life. These attitudes and beliefs are partly based on traditional Chinese culture. For example, In Chinese culture, a wise man knows the meaning and limitations of human life, and the value of integration of oneself with nature. A recent study also related wisdom and life satisfaction in aging people [33]. It was suggested that the awareness and acceptance of human limitations is especially crucial in old age.

Although the psychosocial domain has been thoroughly discussed in the QOL literature, much QOL research and many questionnaires have failed to address a broader set of psychosocial variables, as we have tried to do in this study. Further studies are needed to explore the importance of 'life attitude and retrospection', 'philosophy of living', and 'self-efficacy' on QOL and their relations with other QOL domains.

In the FGI, we observed some individual differences in the preferences of life quality. These differences were more marked in the dimensions of social activity and service, physical environment, leisure activities, religious beliefs, and attitudes towards death.

While QOL issues were quite similar among focus groups, differences were observed between the institutional and community groups. In the institutional group, the elderly emphasized the relationship between the individual and the envi- ronment, and the needs of the elderly person. In the community group, the elderly emphasized the relationship with family members and took the needs of the family as their personal needs. Because most people in the community lived with their families, the well-being of both the family as a whole and individual family members was frequently placed as the first priority. This phenomenon is compatible with Chinese culture and consistent with previous studies in the literature [34]. Elderly people generally give the best or the largest part of their resources to their offspring. Institutionalized elderly people paid more attention to themselves. They emphasized personal needs and experiences, adapted to their own changes, and focused on the relationship between themselves and the environment. However, this does not suggest that family is not important for institutionalized elderly people. Living in institutions may be their last or inevitable choice.

Our findings concerning emphasis on personal needs and experiences supports Lawton's QOL hypothesis that interaction between the person and the environment may have an important role in the subjective evaluation of life quality [35]. Health can be defined as the status of adaptation to the environment [36]. Life quality may be the result of the self-evaluation of personal fitness to the present environment. It was proposed that quality of environment is a major determinant of QOL especially for older people living in an institutional setting [37]. So, it is very possible that the differences between elderly people living in their community or in institutions do not come from different needs but from how they meet their needs. In a community study of 8550 elderly Chinese people in China, researchers found that subjects whose QOL rating ranked lower in objective terms sometimes professed higher satisfaction in subjective terms, and vice versa [38]. In a study of self-rated health in a group of 411 Chinese elderly people living in institutions, the divergence between the global health rating and their health compared with others of the same age was observed in some individuals. More people rated their health 'fair' or 'poor' but 'similar to' or 'better than' others of the same age than rated their health 'average' or 'good' but 'worse' or 'the worst' compared to others of the same age [39]. These findings suggest that an individual's 
perception of health status depends upon the values and reference standards used for comparison.

Cultural differences exist between how Chinese and Western people define the meaning of some QOL dimensions. Vitality, which evaluated lack of energy in the SF-36, is a good example [11]. Lack of energy and many other physical complaints such as malaise and lack of spirit are usually used to express psychological dysfunction in Chinese people. These conditions were what Kleinman called 'neuroasthenia' [40].

In our study, elderly persons expressed their vitality by engaging in social activities, exercise and leisure activities. Our observations also explained the findings of Ren et al. [11]. In their study, two social functioning items were highly correlated with vitality and the mental health scale. Being energetic may not be a norm for Chinese elderly people. In our study, some elderly people stated that people should rest and do nothing when they grow old.

During the cross-cultural adaptation of the SF36 into a Chinese (Taiwanese) version, researchers found that the role-emotional subscale was strongly associated with the physical component and the bodily pain subscale was associated with the mental component in the factor analysis [4]. A similar but weaker association between the bodily pain subscale and the mental component was observed in the test of the SF-36 Hong Kong version [6]. These findings contradicted the original hypothesized association and can be explained by the somatization tendency of Chinese people.

Contrary to our expectations before conducting the focus groups, we found that Chinese elderly people can verbalize their needs, attitudes and internal feelings, and discuss them with other people in a small group. This suggests that we should empower elderly people to take a more active role in their well being.

Shortcomings of this study are the limited number of focus groups and included elderly people only from the northern part of the island. With only a small number of groups and lack of heterogeneity in the elderly people recruited, it is possible that the information we collected did not reach theoretical saturation. Given the opportunity, it would be beneficial to expand this research to include more focus groups, especially with elderly people from other parts of Taiwan (the central and southern parts of the island), and to include minority groups, those with poor health status, and those in low socioeconomic classes. Since group members were recruited in one residential home and one neighborhood, it is possible that established communication styles and relationships among study participants interfered with the data from the focus group process. Fortunately, there were no family members, close friends or superior-subordinate relationships among participants, and all group members worked well on an equal basis.

In summary, our study suggests that the FGI can be a practical method of gathering information from elderly Chinese people from Taiwan even though Chinese people were formerly thought to have difficulty in verbalizing their feelings. Positive life domains as well as negative life domains are equally important to elderly people. Person-environment interaction is a major variable in the evaluation of QOL in elderly people. Family ties are an important component of QOL. Traditional Chinese culture exerts a positive influence on elderly people by providing guidance in dealing with the process of aging. Although most QOL domains are similar between the Western and Chinese elderly, cultural differences exist in several QOL domains such as social functioning, family relationships, and life attitudes as is the case for Chinese living on Mainland China [7, 8]. These differences can be explained by Confucian philosophy, Chinese social structure, the ways Chinese people express emotion and somatization tendency.

The present findings alone may not be sufficient to guide QOL instrument construction and further focus group studies should be done. Further study is needed to determine whether elderly Taiwanese from rural areas, with poor health status, and in low socioeconomic classes have different QOL domains or emphasize different components of QOL from those found in this study.

\section{Acknowledgements}

This study was supported by grant DOH83-HP52-5M01 from the Department of Health, Taiwan, ROC. We thank Hsiu-Jung Chen, Yao-Cheng Lin, Chung-Hsin Chiang, Chien-Hsi Hsu, and Jen- 
Chao Hsiao for their assistance in leading the focus groups.

\section{References}

1. Carver DJ, Chapman CA, Thomas VS, Stadnyk KJ, Rockwood K. Validity and reliability of the Medical Outcomes Study Short Form-20 questionnaire as a measure of quality of life in elderly people living at home. Age Ageing 1999; 28(2): 169-174.

2. De Leo D, Diekstra RF, Lonnqvist J, et al. LEIPAD, an internationally applicable instrument to assess quality of life in the elderly. Behav Med 1998; 24(1): 17-27.

3. Kutner NG, Ory MG, Baker DI, Schechtman KB, Hornbrook MC, Mulrow CD. Measuring the quality of life of the elderly in health promotion intervention clinical trials. Public Health Rep 1992; 107: 530-539.

4. Fuh JL, Wang SJ, Lu SR, Juang KD, Lee SJ. Psychometric evaluation of a Chinese (Taiwanese) version of the SF-36 health survey amongst middle-aged women from a rural community. Qual Life Res 2000; 9: 675-683.

5. Zhao H, Kanda K. Translation and validation of the Standard Chinese version of the EORTC QLQ-C30. Qual Life Res 2000; 9: 129-137.

6. Lam CLK, Gandek B, Ren XS, Chan MS. Test of scaling assumptions and construct validity of the Chinese (HK) version of the SF-36 health survey. J Clin Epidemiol 1998; 51: 1139-1147.

7. Tao G, Chen F, Liu X, et al. Analysis of related factors to life satisfaction of 1380 old people in Beijing. Chinese Mental Health J 1998; 12: 338-340 [Chinese].

8. Zhou Y, Zhu Z, Zhong S. A study on the relationship of primary hypertension in elderly and their quality of life in Changsha City. Chinese J Epidemiol 1998; 19: 24-26 [Chinese].

9. Yang DS, Li LJ, Zhang YL, et al. Study on quality of life of general population in Human province: Part I. Theoretical framework. Chinese Mental Health J 1995; 9: 136139 [Chinese].

10. Li LJ, Hao W, Yang DS, et al. Study on quality of life of general population in Hunan province: Part III. Development of the Quality of Life Inventory. Chinese Mental Health J 1995; 9: 227-231 [Chinese].

11. Ren XS, Amick III B, Zhou L, Gandek B. Translation and psychometric evaluation of a Chinese version of the SF-36 Health Survey in the United States. J Clin Epidemiol 1998; 51: 1129-1138.

12. Ip WC, Kwan YK, Pong GTY, Poon ACK. A health status indicator for the Chinese elderly in Hong Kong. Int BioMed Comput 1995; 40: 133-137.

13. Molassiotis A, Chan CW, Yam BM, Chan SJ. Quality of life in Chinese women with gynaecological cancers. Support Care Cancer 2001; 9(1): 18-24.

14. Phillips DR (ed.), Ageing in east and south-east Asia. London: Edward Arnold, 1992.

15. Yu CLM, Fielding R, Chan CLW, et al. Measuring quality of life of Chinese cancer patients: A validation of the Chi- nese version of the Functional Assessment of Cancer Therapy General (FACT G) Scale. Cancer 2000; 88: 17151727.

16. Cheng AY, Tsui EY, Hanley AJG, Zinman B. Cultural adaptation of the diabetes quality of life measure for Chinese patients. Diabetes Care 1999; 22: 1216-1217.

17. Ho SC, Woo J, Lau J. Life satisfaction and associated factors in older Hong Kong Chinese. J Am Geriatr Soc 1995; 43: 252-255.

18. Lam CLK, Lauder IJ. The impact of chronic diseases on the health-related quality of life (HRQOL) of Chinese patients in primary care. Fam Pract 2000; 17: 159-166.

19. Ren XS, Chang K. Evaluating health status of elderly Chinese in Boston. J Clin Epidemiol 1998; 51: 429-435.

20. Chang DF, Chun CA, Takeuchi DT, Shen H. SF-36 Health Survey: Tests of data quality, scaling assumptions, and reliability in a community sample of Chinese Americans. Med Care 2000; 38: 542-548.

21. Directorate-General of Budget, Accounting \& Statistics. A Study on the Youth and the Elderly in Taiwan. Taipei: DGBAS, Executive Yuan, ROC, 1987.

22. Directorate-General of Budget, Accounting \& Statistics. Report on the old status survey Taiwan area, Republic of China. Taipei: DGBAS, Executive Yuan, ROC, 1992.

23. Basch CE. Focus group interview: An underutilized research technique for improving theory and practice in health education. Health Educ Q 1987; 14: 411-448.

24. Calder BJ. Focus groups and the nature of qualitative research. J Market Res 1977; 14: 353-364.

25. Krueger RA. Focus Groups: A Practical Guide for Applied Research. California, Sage, 1994.

26. Lerner DJ, Levine S. Health-related quality of life: Origins, gaps, and directions. In: Fitzpatrick R (ed.), Advances in Medical Sociology: Quality of Life in Health Care. Connecticut: JAI press, 1994; 139-158.

27. Gilson BS, Gilson JS, Bergner M, et al. The Sickness Impact Profile: Development of an outcome measure of health care. Am J Public Health 1975; 65: 1304-1310.

28. Patrick DL, Erickson P. Assessing health-related quality of life for clinical decision-making. In: Walker SR, Rosser RM (eds), Quality of Life Assessment: Key Issues in the 1990s. Dordrecht: Kluwer Academic, 1993; 11-63.

29. Bandura A. Self-efficacy: Toward a unifying theory of behavioral change. Psychol Rev 1977; 84: 191-215.

30. Grembowski D, Patrick D, Diehr P, et al. Self-efficacy and health behavior among older adults. J Health Soc Behav 1993; 34(2): 89-104.

31. Hess BB, Waring JM. Changing patterns of aging and family bonds in later life. Fam Coordinator 1978; 27: 303314.

32. Venkatraman MM. A cross-cultural study of the subjective well-being of married elderly persons in the United States and India. J Gerontol 1995; 50B: S35-S44.

33. Ardelt M. Wisdom and life satisfaction in old age. J Gerontol 1997; 52B: P15-P27.

34. Best DL, Williams JE. Sex, gender, and culture. In: Berry JW, Segall MH, Kagitcibasi C (eds), Handbook of CrossCultural Psychology. 2nd ed. vol 3. Boston: Allyn \& Bacon, 1980; 163-212. 
35. Lawton MP. Environment and other determinants of wellbeing in older people. Gerontologist 1983; 23: 349-357.

36. Wylie CM. The definition and measurement of health and disease. Public Health Rep 1970; 85: 100-104.

37. Ward P. Quality of Life in Residential Care. London: Personal Social Services Council, 1980.

38. Li L, Young D, Wei H, et al. The relationship between objective life status and subjective life satisfaction with quality of life. Behav Med 1998; 23: 149-159.

39. Leung KK, Tang LY, Lue BH. Self-rated health and mortality in Chinese institutional elderly persons. J Clin Epidemiol 1997; 50: 1107-1116.
40. Kleinman A. The cultural meanings and social uses of illness: A role for medical anthropology and clinically oriented social science in the development of primary care theory and research. J Fam Pract 1983; 16: 539-545.

Address for correspondence: Kai-Kuen Leung, Department of Family Medicine, National Taiwan University Hospital, No. 1 Chang-Te St., Taipei, 10016, Taiwan, ROC

Fax: + 886-2-23118674

E-mail: kkleung@ha.mc.ntu.edu.tw 